\title{
Target Motion Estimation Via Multistatic Forward Scatter Radar
}

\author{
M.Contu*, D. Pastina*, P. Lombardo* \\ A. De Luca**, M. Gashinova**, L. Daniel**, M. Cherniakov** \\ *DIET - University of Rome "La Sapienza" \\ Via Eudossiana, 18 - 00184 Rome, Italy \\ contu@diet.uniroma1.it \\ $\{$ lombardo, debora\}@infocom.uniroma1.it \\ **EECE, University of Birmingham, \\ Birmingham B15 2TT, UK \\ m.s.gashinova@bham.ac.uk
}

\begin{abstract}
The focus of this paper is on the estimation of the kinematic parameters of moving targets via a MIMO Forward Scatter Radar (FSR) system. A sub-optimum estimation technique is considered that exploits the information concerning the time instants at which the target crosses the individual baselines to retrieve the motion parameters. The accuracy of such technique is firstly investigated from a theoretical point of view and then the effectiveness of the proposed approach is demonstrated by applying it to live MIMO FSR data. Shown results prove the practical applicability of the proposed technique.
\end{abstract}

\section{Introduction}

Phenomenological and practical aspects of a single node FSR have been largely studied in [1] and optimized signal processing for target detection and target motion parameters estimation has been devised for this case, as in [2]. It is known the special appeal of FS configuration to detect and localize low-observable targets with negligibly backscatter RCS through their shadow response. In addition in Mie and optical scattering region a forward scatter effect greatly enhances the target detectability [1].

Although target localization with the single RF link is analyzed in [3] it is essential to use multiple FSR nodes for a reliable and accurate estimation of all target motion parameters, especially for the low SNR case. Moreover a reasonable localization accuracy was achieved using the multiple RF links, either in $\mathrm{Rx}$, [4], or Tx, [5], for high SNR cases, where instantaneous Doppler frequency and target crossing angle can be estimated from the received signal.

In [6] the feasibility of joint processing of the signals acquired by a multi-static FSR system for estimation of the target motion parameters was demonstrated assuming orthogonality of the emitted waveforms in frequency domain resulting in an increased robustness in presence of clutter, multipath and interferences. Starting from the results in [6] this paper focuses on the crossing times based target motion estimation technique: such technique exploits the linear relation between the parameters to be estimated (initial position $\left(x_{0}, y_{0}\right)$ and velocity components along the two axes $\left.\left(v_{x}, v_{y}\right)\right)$ and the time instants when the target obscures the direct signal of each couple Tx-Rx. To estimate these parameters at least four baselines crossing times are exploited in a multistatic configuration. The technique advantages are related to the easier implementation and lower computational load. Its performance is theoretically investigated in terms of bias and accuracy under different signal to noise conditions showing unbiased and highly accurate estimated values. Moreover the effectiveness of the proposed approach is further demonstrated by applying it to live MIMO FSR data.

The paper is organized as follows: after briefly recalling the MIMO FSR system features and signal model (Section 2) in Section 3 we recall the considered estimation technique and analyze its theoretical performance. Section 4 is devoted to the presentation and discussion of results obtained by processing the live MIMO FSR data while some conclusions are drawn in Section 5. 


\section{MIMO FS radar received signal model}

We assume two parallel arrays consisting of $N$ transmitters $\left\{\mathrm{T}_{0}, \ldots, \mathrm{T}_{\mathrm{N}-1}\right\}$ and $K$ receivers $\left\{\mathrm{R}_{0}, \ldots, \mathrm{R}_{\mathrm{K}-1}\right\}$, equidistantly distributed with the same spacing $d$, and aligned along a direction orthogonal to the $\mathrm{T}_{0^{-}} \mathrm{R}_{0}$ baseline of overall length $D$ (Figure 1). The coordinate system is taken to be centered in $R_{0}$, with the receivers placed along the $x$ axis, while the transmitter $T_{0}$ lies on the $y$ axis, in position $(0, D)$. Each transmitter emits a $\mathrm{CW}$ probing signal of $\lambda_{\mathrm{n}}$ wavelength with appropriate frequency separation from other transmit signals.

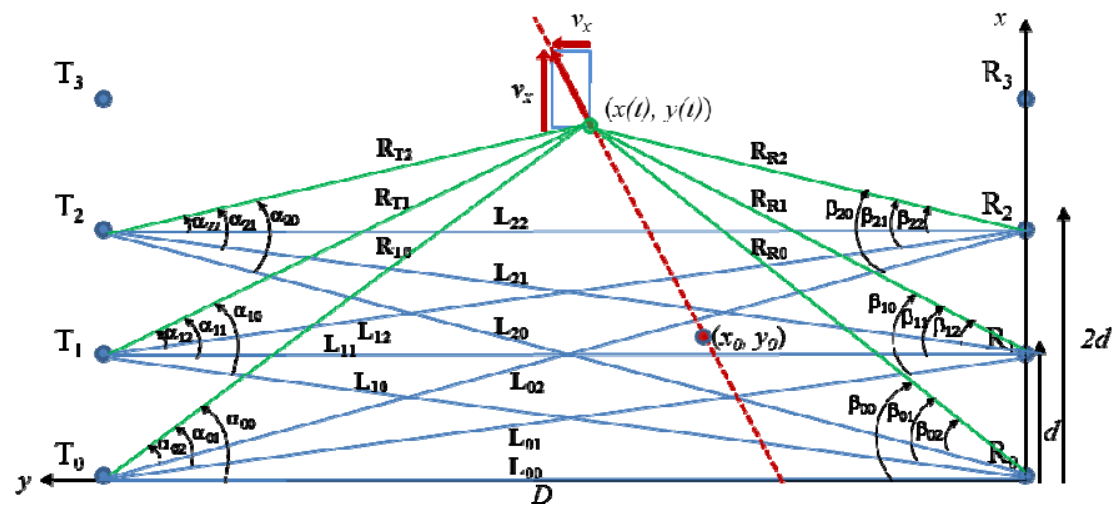

Figure 1: MIMO FS radar geometry - top view.

At this stage for the point-like target we can easily evaluate the relevant geometrical parameters for the generic pair $(n, k)$, of $n$-th transmitter $\left(T_{n}\right) \quad$ and $k$-th receiver $\quad\left(R_{k}\right)$. Baseline of the $T_{n^{-}}$ $R_{k}$ node is given by $L_{n k}=\sqrt{D^{2}+(k-n)^{2} d^{2}}$.

We consider a point-like target moving with the constant velocity so that its coordinates change with time as $x(t)=x_{0}+v_{x} t$ and $y(t)=y_{0}+v_{y} t$. Such a target crosses the baseline $(n, k)$ at a time:

$$
t_{n k}^{0}=\frac{\left(x_{0}-k d\right) D-y_{0}(n-k) d}{v_{y}(n-k) d-v_{x} D}
$$

which we will refer as the $(n, k)$-node Crossing Times (CTs).

For a point-like target we can assume that the phase shift of the scattered signal with respect to (w.r.t.) the direct path signal is defined by the path difference which is the sum of the Tx-target distance and the target-Rx distance, $R_{T n}=\sqrt{(D-y)^{2}+(x-n d)^{2}}$, $R_{R k}=\sqrt{y^{2}+(x-k d)^{2}}$, subtracted by the baseline. Therefore, a target with a velocity component along $x$ (velocity component normal to the Tx-Rx baseline) has a significant nonlinear, generally quadratic, phase term with time.

As first approximation, the amplitude of the EM field scattered by the low-observable target can be assumed to be dependent only on its "shadow aperture", which basically represents set of secondary sources distributed within the area outlined by the shadow profile, but not on the target material or shape. This provides a non-negligible scattered field intensity even for dielectric objects with shapes designed to be "stealth" for a backscatter sensing. Approximating the target "shadow aperture" by a rectangular shape of dimensions $l_{h}$, (horizontal) and $l_{v}$ (vertical) the combination of the direct signal with the forward scattered signal is:

$$
U_{n k}^{T O T}=A e^{j \frac{2 \pi}{\lambda_{n}} L_{n k}}\left(1+j \frac{l_{v} l_{h} \cdot L_{n k}}{\lambda_{n} R_{T n} R_{R k}} \cdot e^{j \frac{2 \pi}{\lambda_{n}}\left[R_{T n}+R_{R k}-L_{n k}\right]} \cdot \varepsilon_{n k}(x, y)\right)
$$

where $A$ is the amplitude of the leakage signal and the amplitude of the signal scattered by target in a forward direction is modulated by the quantity $\varepsilon_{n k}(x, y)$ that changes with time due to the target motion (see [6]) and depends from geometrical parameters $\alpha_{n n}$ and $\beta_{k k}$ defined in Figure 1 , and $\varphi$ is the baseline crossing angle. Finally white Gaussian thermal noise is added to ( 2 ) providing the received signal given by $r_{n, k}(t)=U_{n, k}^{T O T}(t)+n_{n, k}(t)=S_{d_{n, k}}+S_{t_{n, k}}(t)+n_{n, k}(t)$. 


\section{Motion Parameter Estimation}

The approach considered for motion parameters estimation is based on the exploitation of solely the multi-node CTs: for this reason it is expected to be robust to multipath interference and clutter but requires the availability of multiple baselines. As derived in [6] the unknowns are evaluated as a solution of a linear system of at least 4 equations (one for each node $\left(n_{p}, k_{p}\right)$ with $P \geq 4, p=1, \ldots, P$ ) by means of the following equation:

$$
\left[\begin{array}{c}
x_{0} \\
y_{0} \\
v_{x} \\
v_{y}
\end{array}\right]=\left(\mathbf{C}^{T} \mathbf{C}\right)^{-1} \mathbf{C}^{T}\left[\begin{array}{c}
k_{1} \\
k_{2} \\
k_{3} \\
\vdots \\
k_{P}
\end{array}\right] d
$$

As apparent one of the main tasks of the estimation procedure is to extract the CTs to correctly build matrix $\mathbf{C}$ : the accuracy of the used $t_{n, k}$ values will impact on the estimation accuracy of the unknowns. Theoretical performance achievable in target motion parameters estimation is investigated here for three different approaches.

In the first approach the extracted CT are independent Gaussian random variables with mean value set to eq. ( 1 ) and standard deviation given by the Cramer-Rao lower bound for time delay [7] $\sigma_{n, k}=\frac{1}{\beta_{n, k} \cdot \sqrt{S N R_{n, k}}}$ where $\beta_{n, k}$ is the effective bandwidth of the baseline $(\mathrm{n}, \mathrm{k})$ target signal and $S N R_{n, k}$ is the Signal to Noise Ratio of the same baseline evaluated after integration.

In the second approach the received signal $r_{n, k}(\mathrm{t})$ is processed by a Maximum Likelihood (ML) processor and the time instant providing the highest peak at the processor output is retained as the estimated crossing time. The ML detector can be proven equal to:

$$
\sum_{m=1}^{M} 2 \Re e\left\{\left[r_{n, k}(m)-S_{d_{n, k}}(m)\right] \cdot S_{t_{n, k}}^{*}(m)\right\}
$$

being $M$ the number of samples in the processed interval. ML strategy is equivalent to cancel out the leakage signal and process the cancelled signal with a filter matched to $S_{t}$ (target component).

The last approach is the non-coherent (NC) technique, where the processed signal is the received signal filtered by a square law detector: $d_{n, k}(t)=\left|r_{n, k}(t)\right|^{2}=d_{D S_{n, k}}(t)+d_{D H_{n, k}}(t)$, being $d_{D S_{n, k}}(t)$ and $d_{D H_{n, k}}(t)$ the direct signal contribution and the information about the target Doppler history respectively. The detector in (5) consists of a cross-correlation with a DC removal stage to suppress both the direct signal component and the continuous component resulting from the filtering of the AWG noise with the square law detector.

$$
\sum_{m=1}^{M}\left\{\left[\left(d_{S_{n, k}}(m)-d_{D S_{n, k}}(m)\right)-\left\langle d_{S_{n, k}}(m)-d_{D S_{n, k}}(m)\right\rangle\right] \cdot d_{D H_{n, k}}(m)\right\}
$$

Focusing on the theoretical performance the perfect knowledge of both the leakage signal $S_{d_{n, k}}$ and the target signal $S_{t_{n, k}}$ is here assumed.

The performance of the CT-based estimation technique is investigated via Monte Carlo simulations in terms of bias and accuracy as functions of the integrated signal to noise power ratio $S N R_{n, k}$ related to the direct signal to noise power ratio $D N R_{n, k}$ by the following: $S N R=2 D N R \sum_{m=1}^{M}\left|S_{t_{n, k}^{\prime}}^{\prime}(m)\right|^{2}$ where $S_{t_{n, k}}^{\prime}(m)$ is the normalized target signal contribution. The parameters related to the MIMO FSR system and to the target motion used in the simulated analysis have been set to values scaled of a factor 10 w.r.t. the experimental trials described in Section 4. Particularly the same small target size (of area $0.243 \mathrm{~m}^{2}$ ) is considered whereas initial position is given by $(0,50) \mathrm{m}$ and the target velocity is given by $(12,0) \mathrm{m} / \mathrm{s}$. 
The simulated analysis proved that the technique is able to provide unbiased values for both initial position and velocity. The maximum achievable theoretical accuracy (dot-dashed curves) compared to the accuracy achievable by extracting the CTs via a ML approach (continuous curves) and via the $\mathrm{NC}$ detector (dashed curves) is shown in Figure 2.a and Figure 2.b.
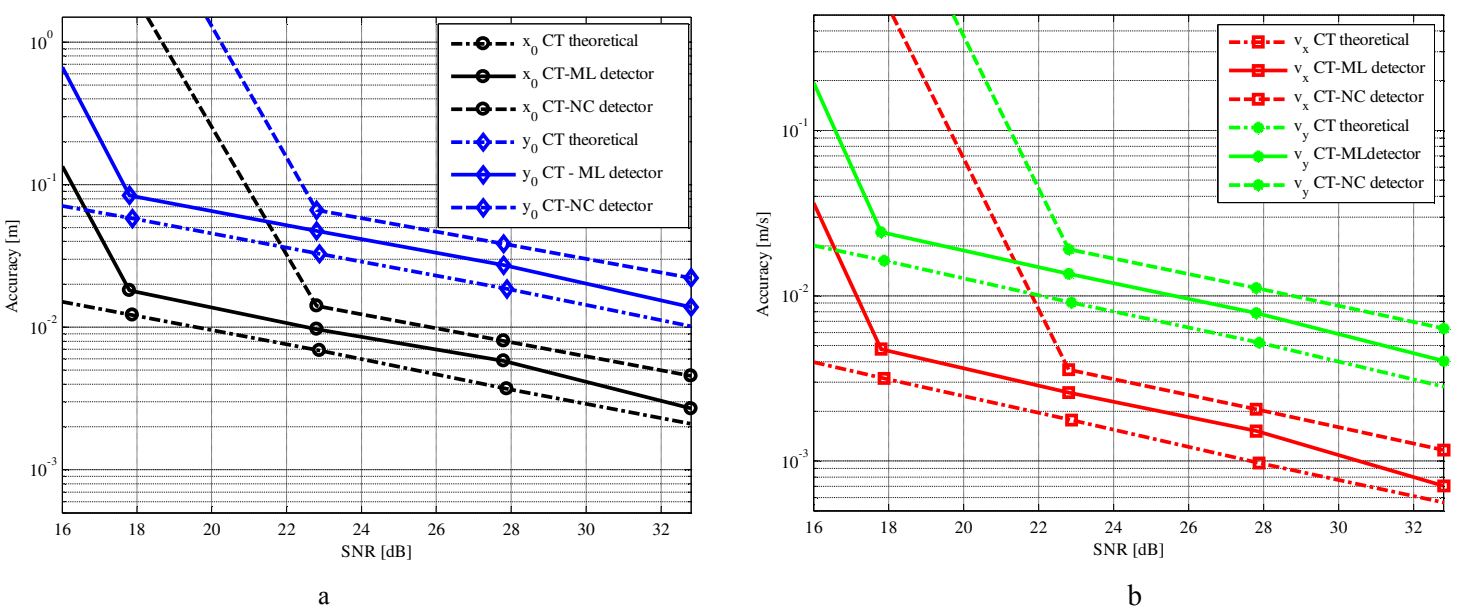

b

Figure 2:Standard deviation of the estimation error on the initial position (a) and velocity components (b).

Apparently, for each parameter to be estimated, the dot-dashed and continuous curves are very close in the medium-to-high values of SNR region: some losses w.r.t. the highest achievable accuracy are experienced for very low values of the integrated SNR (i.e. SNR before integration $-3.9 \mathrm{~dB}$ ). As expected the accuracy achievable for $v_{x}$ (the component parallel to the Tx/Rx arrays) is by far better than for $v_{y}$. Moreover it can be observed that the accuracy provided by the NC approach shows a degradation w.r.t. the ML technique since the phase and quadrature signals information is no longer exploited. In particular NC detector shows a threshold value of integrated SNR of $5 \mathrm{~dB}$ more than ML whereas for higher SNR values the curves show the same trend with some light extra losses experienced by the NC technique. In any case, apart from very low non-integrated SNR, both initial position and target speed are accurately estimated thus theoretically proving effectiveness of the proposed approach.

\section{Application to live recorded MIMO FSR data}

An experimental setup has been developed at the University of Birmingham composed of 2 Tx and $4 \mathrm{Rx}$, providing 8 different Doppler profiles. Two Txs transmit Gaussian shaped RF pulses of $10 \mathrm{MHz}$ frequency bandwidth and a PRF of $1 \mathrm{kHz}$ at carrier frequencies of $3.1 \mathrm{GHz}$ and $3.4 \mathrm{GHz}$, respectively, using horn antennas elevated $0.35 \mathrm{~m}$ above the ground. Four Rxs use omni-directional antennas at a height of $0.25 \mathrm{~m}$. The system geometry has been set as a compromise to satisfy the fundamental operation conditions: optical scattering region and far field operation. In this dataset, true values of the initial target position and velocity can be assumed to be $(0,5) \mathrm{m}$ and $\mathbf{v}=(1.2,0) \mathrm{m} / \mathrm{s}$ respectively, in agreement with the case study of Section 3.

As shown in Figure 3 the target Doppler signature corresponding to $90^{\circ}$ crossing angle has a shape that can be significantly affected by multipath or interference, whereas the center of the Doppler signature is stable enough to provide CTs. 


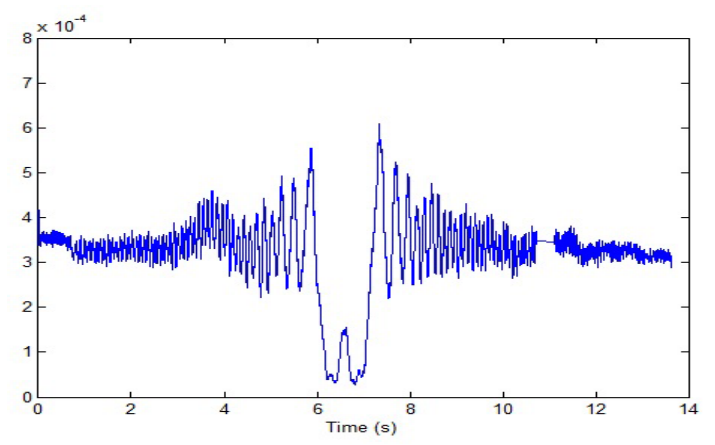

Figure 3: Target signature example.

Table I shows the CTs values obtained for the eight baselines by applying the NC CTs extraction technique (as in (5)). Results in column $a$ are obtained using as reference target signal for cross correlation a windowed signal extracted from the recorded signal in noise free conditions $(2.7 \mathrm{~s}$ centered in the baseline $\mathrm{CT}$ as in Section 3), whereas results of column $b$ are obtained with a synthetic signal generated in agreement with the model presented in Section 2 as reference signal. Assuming a priori knowledge on the target motion parameters the processing via a database of synthetic waveforms matching to a range of trajectory parameters is emulated. Third column shows the correlation coefficient $\rho_{n k}$ among the two reference signals used in cases $a$ and $b$. It allows evaluating whether similar performance can be expected from different approaches.

Table I: estimated CTs and cross-correlation coefficients between reference signals in case $a$ and case $b$.

\begin{tabular}{|c|c|c|c|}
\hline \multirow{2}{*}{$\begin{array}{c}\text { Baseline } \\
(\mathrm{n}, \mathrm{k})\end{array}$} & \multicolumn{2}{|c|}{$\begin{array}{c}\text { Crossing Times } \\
t_{n, k}[\mathrm{~s}]\end{array}$} & \multirow{2}{*}{$\rho_{n, k}$} \\
\cline { 2 - 3 } & $a$ & $b$ & \\
\hline$(1,1)$ & -2.4770 & -2.4910 & 0.6000 \\
\hline$(1,2)$ & -1.3690 & -1.3480 & 0.5692 \\
\hline$(1,3)$ & -0.2610 & -1.0010 & 0.0278 \\
\hline$(1,4)$ & 0.8510 & 0.7890 & 0.7048 \\
\hline$(2,1)$ & -0.8120 & -0.7550 & 0.4548 \\
\hline$(2,2)$ & 0.2980 & 0.3510 & 0.5889 \\
\hline$(2,3)$ & 1.4070 & 1.4420 & 0.5338 \\
\hline$(2,4)$ & 2.5150 & 2.4680 & 0.6143 \\
\hline
\end{tabular}

Table II: estimated kinematic parameters with the two approach $a$ and $b$

\begin{tabular}{|c|c|c|c|c|c|}
\hline \multirow{2}{*}{$\begin{array}{c}\text { Kinematic } \\
\text { parameters }\end{array}$} & \multicolumn{5}{|c|}{ CT based estimation technique } \\
\cline { 3 - 6 } & Case $a$ & $L=0$ & $L=1$ & $L=2$ & $L=4$ \\
\cline { 3 - 6 } & -0.0226 & 0.1390 & -0.0232 & -0.0231 & -0.0245 \\
\hline $\mathbf{x}_{\mathbf{0}}[\mathrm{m}]$ & 4.9973 & 5.4496 & 4.9962 & 4.9967 & 4.9937 \\
\hline $\mathbf{y}_{\mathbf{0}}[\mathrm{m}]$ & 1.1998 & 1.1394 & 1.2000 & 1.1999 & 1.2004 \\
\hline $\mathbf{v}_{\mathbf{x}}[\mathrm{m} / \mathrm{s}]$ & -0.0010 & -0.3826 & 0.0001 & -0.0003 & 0.0034 \\
\hline $\mathbf{v}_{\mathbf{y}}[\mathrm{m} / \mathrm{s}]$ & & & & &
\end{tabular}

Obviously when the correlation coefficient is high the extracted CT values are quite similar to each other. Unfortunately some baselines have poor $\rho_{n k}$ values which are unsuitable for the estimation purpose (e.g. baseline $(1,3)$ ). This mismatching between the recorded signal and the modeled signal is due to both: first, not strict validity of the assumptions of target being in optical scattering region and far field operation and, second, the corruption of the target signatures by interference and multipath. Obviously the kinematic parameters estimation by means of the proposed CT-based estimation technique will suffer of this error in CTs estimation. Results shown in Table II confirm our considerations. In case $a$ the estimated values are completely in agreement with the true values, whereas in case $b$, if all baseline CTs are used jointly $(L=0)$ some degradation is observed. Note that discarding estimated CTs which suffer from the missmatching degradation, using the correlation coefficient as a quality indicator, we obtain better performance. Different cases are investigated where only the $P$ - $L$ best baselines (with $L=1,2,4$ ) are considered, consequently reducing the dimensionality of matrix $\mathbf{C}$ in Eq. (3). As shown in Table II a sensible improvement in the estimated values is obtained excluding the worst baseline $(L=1)$ resulting in estimated parameters values by far closer to those of case $a$, w.r.t. those obtained for $L=0$. For $L=2$ slightly better performance are obtained, whereas a degradation is appreciable with $L=4$, thus confirming the gradual worsening due to the exploitation of less 
baselines information. To further confirm the validity of the proposed approach, Figure 4 shows the accuracy of the estimated position, (a), and speed, (b), as a function of the integrated SNR obtained as for the results of $\mathrm{NC}$ detector in Figure 2. The two different approaches already described in cases $a$ and $b$ have been studied. As we can see the data based (continuous line) results in Figure 4 are largely in agreement with those corresponding to dashed lines in Figure 2 and considerations similar to those already done for plots in Section 3 apply. Moreover comparing results obtained in case $a$ and $b$ with seven baselines, it is clear that the curves has the same trend whereas the accuracy values suffers of moderate losses due to both the miss-matching of the residual baselines and the completely missing information of the discarded baseline. Finally looking at the dotted curves obtained by using minimum number of baseline $(L=4)$ we see that accuracies get even worse, as we expected from the preliminary considerations discussing data in Table II.
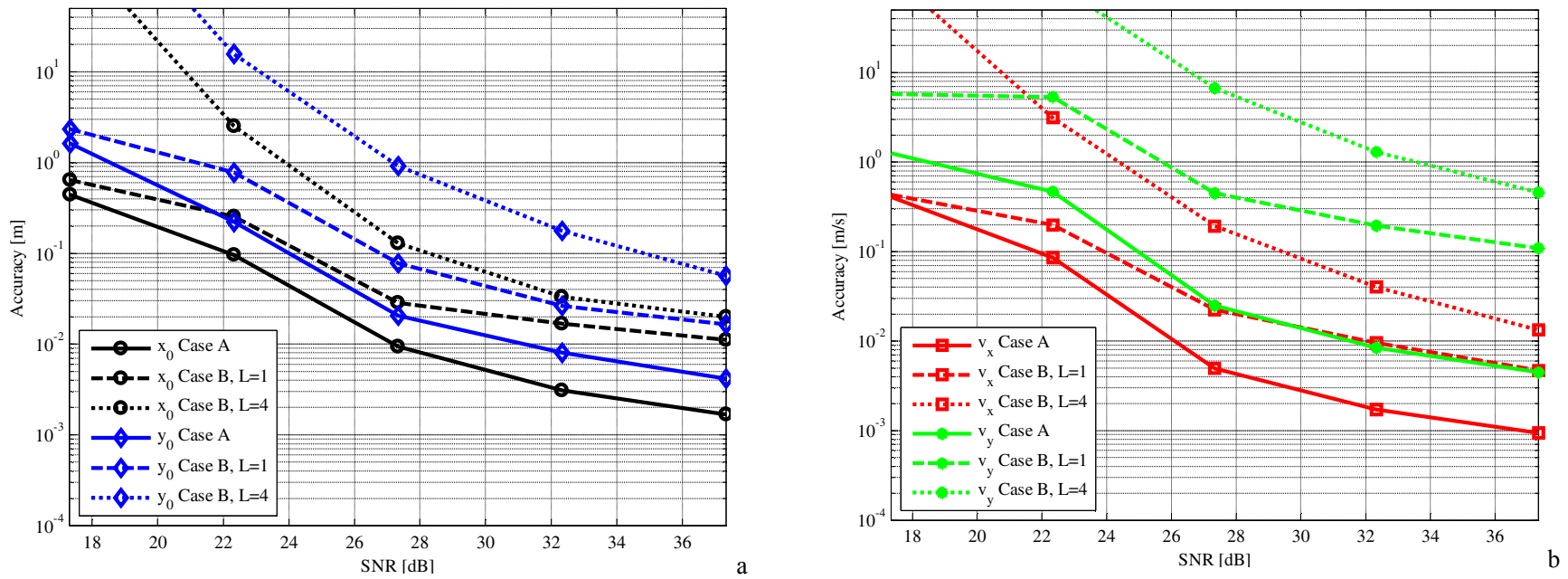

Figure 4: Accuracy versus integrated SNR for experimental data: initial position (a) and velocity components (b).

\section{Conclusions}

A CT based estimation technique has been proposed to estimate the kinematic parameters of lowobservable targets moving in the area surveyed by a MIMO FSR system and its performance has been theoretically investigated. The analysis proved that unbiased estimates of initial positions and speed components are obtained with high accuracy even in poor SNR conditions. Theoretical performance has also been validated by processing data acquired by an experimental MIMO FSR system. Shown results prove the practical applicability of the technique and demonstrate the potential of MIMO FSR.

\section{References}

[1] M. Gashinova, L. Daniel, K. Kabakchiev, V. Sizov, E. Hoare, M. Cherniakov et al. "Phenomenology of Doppler forward scatter radar for surface targets observation" ,IET RSN, vol.7, no.4, pp.422-432, 2013

[2] H. Cheng; V. Sizov; M. Antoniou; M. Gashinova; M. Cherniakov; "Optimal Signal Processing in GroundBased Forward Scatter Micro Radars," IEEE TAES, v.48, no.4, pp.3006-3026, October 2012

[3] A. Blyakhman and I. Runova, "Forward scattering radiolocation bistatic RCS and target detection", IEEE International Radar Conference, Waltham, MA, Apr. 1999, pp. 203-208.

[4] A. Blyakhman; A. Ryndyk and S. Sidorov, "Forward scattering radar moving object coordinate measurement", IEEE International Radar Conference, Alexandria, VA, May 2000, pp. 678-82.

[5] A.G. Ryndyk, A.A Kuzin, A.V. Myakinkov, "Target tracking in forward scattering radar with multi-beam transmitting antenna", IEEE International Radar Conference, 2009, pp. 1-4.

[6] M. Gashinova, L Daniel, M Cherniakov, P.Lombardo, D.Pastina, A. De Luca, "Multistatic Forward Scatter Radar for accurate motion parameters estimation of low observable targets" IEEE International Radar Conference, Lille, France, October 2014.

[7] M. Skolnik, Radar Handbook, Third edition, Mc Graw Hill. 